Proceedings of the Edinburgh Mathematical Society (2006) 49, 131-143 (C)

DOI:10.1017/S0013091504000306 Printed in the United Kingdom

\title{
NORM ESTIMATES OF THE PRE-SCHWARZIAN DERIVATIVES FOR CERTAIN CLASSES OF UNIVALENT FUNCTIONS
}

\author{
YONG CHAN KIM ${ }^{1}$ AND TOSHIYUKI SUGAWA ${ }^{2}$ \\ ${ }^{1}$ Department of Mathematics Education, Yeungnam University, \\ 214-1 Daedong, Gyongsan 712-749, Korea (kimyc@yu.ac.kr) \\ ${ }^{2}$ Department of Mathematics, Graduate School of Science, Hiroshima University, \\ Higashi-Hiroshima 739-8526, Japan (sugawa@math.sci.hiroshima-u.ac.jp)
}

(Received 28 April 2004)

\begin{abstract}
A sharp norm estimate will be given to the pre-Schwarzian derivatives of close-to-convex functions of specified type. In order to show the sharpness, we introduce a kind of maximal operator which may be of independent interest. We also discuss a relation between the subclasses of close-to-convex functions and the Hardy spaces.
\end{abstract}

Keywords: pre-Schwarzian derivative; uniformly locally univalent; close-to-convex function

2000 Mathematics subject classification: Primary 30C45

Secondary 30A32

\section{Introduction and definitions}

Let $\mathcal{A}$ denote the class of functions of the form

$$
f(z)=z+\sum_{n=2}^{\infty} a_{n} z^{n}
$$

which are analytic in the open unit disc $\mathbb{D}=\{z \in \mathbb{C}:|z|<1\}$. Also let $\mathcal{S}, \mathcal{S}^{*}$ and $\mathcal{K}$ denote the subclasses of $\mathcal{A}$ consisting of functions which are univalent, starlike and convex in $\mathbb{D}$, respectively. Here, $f \in \mathcal{A}$ is said to be starlike (convex) if $f$ is univalent and if the image $f(\mathbb{D})$ is starlike with respect to 0 (convex). See $[\mathbf{3}]$ for further information on those classes. For analytic functions $g$ and $h$ in $\mathbb{D}, g$ is said to be subordinate to $h$ if there exists an analytic function $\omega$ such that $\omega(0)=0,|\omega(z)|<1$ and $g(z)=h(\omega(z))$ for $z \in \mathbb{D}$. The subordination will be denoted by $g \prec h$, or, conventionally, $g(z) \prec h(z)$. In particular, when $h$ is univalent, $g \prec h$ if and only if $g(0)=h(0)$ and if $g(\mathbb{D}) \subset h(\mathbb{D})$.

We now introduce the terminology needed below. Let $\mathcal{M}$ be the class of non-vanishing analytic functions $\varphi$ in $\mathbb{D}$ with the normalization condition $\varphi(0)=1$. Following Ma and Minda [9], we define the subclasses $\mathcal{S}^{*}(\varphi)$ and $\mathcal{K}(\varphi)$ of $\mathcal{A}$ as the sets of functions $f \in \mathcal{A}$ 
of the forms

$$
\frac{z f^{\prime}(z)}{f(z)} \prec \varphi(z)
$$

and

$$
1+\frac{z f^{\prime \prime}(z)}{f^{\prime}(z)} \prec \varphi(z)
$$

respectively, for each $\varphi \in \mathcal{M}$. By definition, it is obvious that $f \in \mathcal{K}(\varphi)$ if and only if $z f^{\prime} \in \mathcal{S}^{*}(\varphi)$. We note that $\mathcal{S}^{*}(\varphi) \subset \mathcal{S}^{*}(\psi)$ and $\mathcal{K}(\varphi) \subset \mathcal{K}(\psi)$ for $\varphi \prec \psi$.

A typical example for $\varphi$ is given by

$$
\varphi_{A, B}(z)=\frac{1+A z}{1+B z}
$$

where $A$ and $B$ are real numbers satisfying $-1 \leqslant B<A \leqslant 1$. Note that the Möbius transformation $\varphi_{A, B}$ maps the unit disc onto the disc (or half-plane) with diameter $((1-A) /(1-B),(1+A) /(1+B))$. The corresponding classes $\mathcal{K}\left(\varphi_{A, B}\right)$ and $\mathcal{S}^{*}\left(\varphi_{A, B}\right)$ have been studied by Janowski $[\mathbf{4}, \mathbf{5}]$ and Silverman and Silvia $[\mathbf{1 1}]$. We note that $\mathcal{S}^{*}=$ $\mathcal{S}^{*}\left(\varphi_{1,-1}\right)$ is the class of starlike functions and $\mathcal{K}=\mathcal{K}\left(\varphi_{1,-1}\right)$ is the class of convex functions.

In this article, we treat classes of analytic functions defined in a similar way to the class of close-to-convex functions. For functions $\varphi, \psi \in \mathcal{M}$, following [8], we denote by $\mathcal{C}(\varphi, \psi)$ the set of all $f$ in $\mathcal{A}$ such that there exists a function $h \in \mathcal{K}(\varphi)$ with

$$
\frac{f^{\prime}}{h^{\prime}} \prec \psi \text {. }
$$

Note that $\mathcal{S}^{*}(\varphi) \subset \mathcal{C}(\varphi, \varphi)$. The class of close-to-convex functions can be included in our framework in the following way. A function $f \in \mathcal{A}$ is called close-to-convex if there exist a convex function $h \in \mathcal{K}=\mathcal{K}\left(\varphi_{1,-1}\right)$ and a real constant $\gamma$ with $|\gamma|<\pi / 2$ such that $\operatorname{Re}\left(\mathrm{e}^{-\mathrm{i} \gamma} f^{\prime} / h^{\prime}\right)>0$ holds in $\mathbb{D}$. The last condition is equivalent to the subordination $f^{\prime} / h^{\prime} \prec \psi_{\gamma}$, where

$$
\psi_{\gamma}(z)=\frac{1+\mathrm{e}^{\mathrm{i} \gamma} z}{1-\mathrm{e}^{-\mathrm{i} \gamma} z}
$$

Therefore, the class $\mathcal{C}$ of close-to-convex functions can be described as the union of $\mathcal{C}\left(\varphi_{1,-1}, \psi_{\gamma}\right)$ over $-\pi / 2<\gamma<\pi / 2$. It is known that $\mathcal{C} \subset \mathcal{S}$ (see $\left.[\mathbf{3}]\right)$. by

The pre-Schwarzian derivative $T_{f}$ of a locally univalent analytic function $f$ is defined

$$
T_{f}(z)=\frac{f^{\prime \prime}(z)}{f^{\prime}(z)}
$$

We also define the norm of $T_{f}$ by

$$
\left\|T_{f}\right\|=\sup _{z \in \mathbb{D}}\left|T_{f}(z)\right|\left(1-|z|^{2}\right) .
$$


It is known that $\left\|T_{f}\right\|<\infty$ if and only if $f$ is uniformly locally univalent, namely, $f$ is univalent in each hyperbolic disc in $\mathbb{D}$ of a fixed radius. Indeed, the radius of univalence can be estimated in terms of $\left\|T_{f}\right\|$. We note that the set $\mathcal{T}_{1}$ of pre-Schwarzian derivatives $T_{f}$ of those functions $f$ in $\mathcal{S}$ which extend to quasiconformal automorphisms of the Riemann sphere can be regarded as a model of the universal Teichmüller space (cf. [16]), in analogy with the Schwarzians. It is also known that $\left\|T_{f}\right\| \leqslant 6$ for $f \in \mathcal{S}$ and that $\left\|T_{f}\right\| \leqslant 4$ for $f \in \mathcal{K}$, and, conversely, for $f \in \mathcal{A},\left\|T_{f}\right\| \leqslant 1$ implies $f \in \mathcal{S}$ (Becker's theorem).

The authors deduced various properties (distortion, growth, growth of the coefficients and so on) of functions $f \in \mathcal{A}$ with $\left\|T_{f}\right\| \leqslant 2 \lambda$ for a fixed number $\lambda>0$, and gave norm estimates for a few classes of univalent functions in $[6]$. The present article is a continuation of that work. The aim of this paper is to give (possibly sharp) norm estimates of the pre-Schwarzian derivative for the class $\mathcal{C}(\varphi, \psi)$.

Theorem 1.1. Let $\varphi, \psi \in \mathcal{M}$ and suppose that $\varphi$ is univalent and the image $\varphi(\mathbb{D})$ is starlike with respect to 1 . Then the inequality

$$
\left\|T_{f}\right\| \leqslant \sup _{|z|<1}\left(1-|z|^{2}\right)\left|\frac{\varphi(z)-1}{z}\right|+\sup _{|z|<1}\left(1-|z|^{2}\right)\left|\frac{\psi^{\prime}(z)}{\psi(z)}\right|
$$

holds for every $f \in \mathcal{C}(\varphi, \psi)$. Moreover, this estimate is sharp if the inequalities

$$
\left|\frac{\varphi(z)-1}{z}\right| \leqslant \frac{\varphi(\varepsilon|z|)-1}{\varepsilon|z|} \text { and }\left|\frac{\psi^{\prime}(z)}{\psi(z)}\right| \leqslant \frac{\psi^{\prime}(\varepsilon|z|)}{\psi(\varepsilon|z|)}
$$

hold simultaneously for all $z \in \mathbb{D}$, where $\varepsilon$ is a unimodular constant.

The estimate in the main theorem can be obtained in a straightforward way. The sharpness, however, requires more careful observations. To this end, we introduce a sort of maximal operator in connection with the Schwarz-Pick lemma in $\S 2$ and deduce basic properties of it. The authors believe that this methodology is efficient in other extremal problems as well. In the forthcoming paper [7], the quantity $\sup _{z \in \mathbb{D}}\left(1-|z|^{2}\right)\left|\psi^{\prime}(z) / \psi(z)\right|$ is investigated for a non-vanishing analytic function $\psi$ in a more systematic way.

The proof of Theorem 1.1 will be given in $\S 3$. We will give some applications of the main theorem in $\S 4$. We also provide some inclusion relations between the class $\mathcal{C}(\varphi, \psi)$ and the Hardy spaces in $\S 5$.

Finally, we mention a couple of related results. Yamashita [15] investigated the norm of pre-Schwarzian derivatives of Gelfer-starlike, Gelfer-convex and Gelfer-close-to-convex functions (see also [14] for Gelfer functions). Recently, Okuyama [10] gave a sharp norm estimate for the class of $\beta$-spiral-like functions.

\section{An extremal problem and the associated maximal operator}

We first introduce an extremal problem and deduce fundamental properties of the adapted maximal operator.

Let us consider the extremal problem: for a given pair of points $z_{0}, w_{0}$ with $\left|w_{0}\right| \leqslant$ $\left|z_{0}\right|<1$, find the maximum of values $\left|\omega^{\prime}\left(z_{0}\right)\right|$ or, more precisely, the region of values 
$\omega^{\prime}\left(z_{0}\right)$, for holomorphic mappings $\omega: \mathbb{D} \rightarrow \mathbb{D}$ with $\omega(0)=0$ and $\omega\left(z_{0}\right)=w_{0}$. A complete solution to this problem was given by Dieudonné in 1931. The following is known as Dieudonné's lemma (see [3, p. 198]).

Lemma 2.1 (Dieudonné). Let $\mathcal{F}$ be the family of analytic functions $\omega$ on the unit disc with $|\omega|<1, \omega(0)=0$ and $\omega\left(z_{0}\right)=w_{0}$, where $z_{0}$ and $w_{0}$ are points in $\mathbb{D}$ with $\left|w_{0}\right| \leqslant\left|z_{0}\right| \neq 0$. Then the set $\left\{\omega^{\prime}\left(z_{0}\right): \omega \in \mathcal{F}\right\}$ is the closed disc centred at $w_{0} / z_{0}$ with radius $\left(\left|z_{0}\right|^{2}-\left|w_{0}\right|^{2}\right) /\left|z_{0}\right|\left(1-\left|z_{0}\right|^{2}\right)$. Furthermore, if $\omega^{\prime}\left(z_{0}\right)$ lies on the boundary of the disc, then $\omega$ has the form

$$
\omega(z)=z \frac{\lambda\left(\left(z-z_{0}\right) /\left(1-\bar{z}_{0} z\right)\right)+\left(w_{0} / z_{0}\right)}{1+\lambda\left(\bar{w}_{0} / \bar{z}_{0}\right)\left(\left(z-z_{0}\right) /\left(1-\bar{z}_{0} z\right)\right)}
$$

for a constant $\lambda$ with $|\lambda|=1$.

In particular, we obtain the sharp inequality

$$
\left|\omega^{\prime}\left(z_{0}\right)\right| \leqslant\left|\frac{w_{0}}{z_{0}}\right|+\frac{\left|z_{0}\right|^{2}-\left|w_{0}\right|^{2}}{\left|z_{0}\right|\left(1-\left|z_{0}\right|^{2}\right)}=K\left(\left|z_{0}\right|,\left|w_{0}\right|\right)
$$

for such a function $\omega$ with equality holding if and only if $\lambda=w_{0}\left|z_{0}\right|^{2} /\left|w_{0}\right| z_{0}^{2}$. Here $K(r, s)$ is given by

$$
K(r, s)=\frac{s}{r}+\frac{r^{2}-s^{2}}{r\left(1-r^{2}\right)}=\frac{s\left(1-r^{2}\right)+r^{2}-s^{2}}{r\left(1-r^{2}\right)}
$$

for $0 \leqslant s \leqslant r<1$ (we set $K(0,0)=1$ ).

By using the function $K(r, s)$, we define a maximal operator on the set $C([0,1))$ of continuous functions on the interval $[0,1)$. For $F \in C([0,1))$, we set

$$
\hat{F}(r)=\max _{0 \leqslant s \leqslant r} K(r, s)|F(s)|, \quad 0 \leqslant r<1,
$$

and we call $\hat{F}$ the maximal function of $F$.

Apart from the obvious subadditivity $(F+G)^{\wedge} \leqslant \hat{F}+\hat{G}$, the following estimates constitute basic properties of the operator $F \mapsto \hat{F}$.

Lemma 2.2. Let $F$ be a continuous function on the interval $[0,1)$. Then

$$
\left(1-r^{2}\right)|F(r)| \leqslant\left(1-r^{2}\right) \hat{F}(r) \leqslant \max _{0 \leqslant s \leqslant r}\left(1-s^{2}\right)|F(s)| .
$$

Proof. First, by the identity

$$
r\left(1-s^{2}\right)-\left[s\left(1-r^{2}\right)+r^{2}-s^{2}\right]=(r-s)(1-r)(1-s),
$$

we obtain the following estimate of the kernel $K(r, s)$ given in $(2.3)$ :

$$
K(r, s) \leqslant \frac{1-s^{2}}{1-r^{2}}
$$

for $0 \leqslant s \leqslant r<1$. Therefore, the right-hand inequality in (2.5) follows. The left-hand one is obvious because $K(r, r)=1$. 


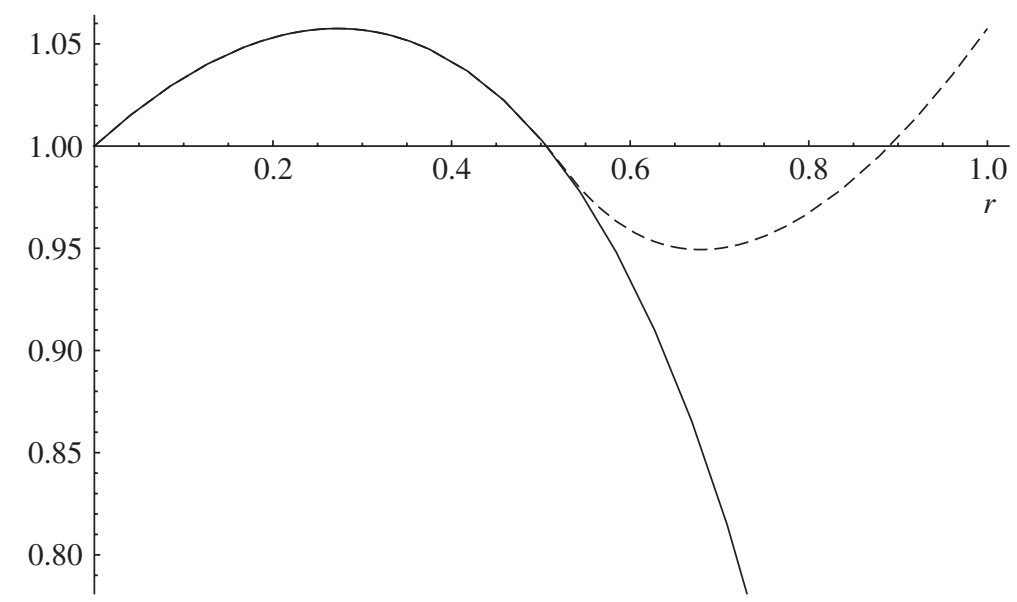

Figure 1. Graphs of $\left(1-r^{2}\right) F(r)$ (solid line) and $\left(1-r^{2}\right) \hat{F}(r)$ (dashed line).

Remark 2.3. In view of the Schwarz-Pick lemma, $\left|\omega^{\prime}(z)\right| \leqslant\left(1-|\omega(z)|^{2}\right) /\left(1-|z|^{2}\right)$ for a holomorphic function $\omega: \mathbb{D} \rightarrow \mathbb{D}$, the inequality (2.6) is a natural conclusion.

As an immediate consequence of the lemma, we obtain the relation

$$
\sup _{0 \leqslant r \leqslant r_{0}}\left(1-r^{2}\right) \hat{F}(r)=\sup _{0 \leqslant r \leqslant r_{0}}\left(1-r^{2}\right)|F(r)|
$$

for any $0 \leqslant r_{0}<1$. In particular,

$$
\sup _{0 \leqslant r<1}\left(1-r^{2}\right) \hat{F}(r)=\sup _{0 \leqslant r<1}\left(1-r^{2}\right)|F(r)| .
$$

We now assume that the supremum of $\left(1-r^{2}\right)|F(r)|$ is attained at $r=r_{0} \in[0,1)$. Then, by Lemma 2.2, we see that $\hat{F}\left(r_{0}\right)=\left|F\left(r_{0}\right)\right|$ and that the supremum of $\left(1-r^{2}\right) \hat{F}(r)$ is attained also at $r=r_{0}$. It is a little surprising that $\left(1-r^{2}\right) \hat{F}(r)$ again tends to the same value as $\left(1-r_{0}^{2}\right) \hat{F}\left(r_{0}\right)$ when $r$ approaches 1 . Figure 1 illustrates the graphs of the functions $\left(1-r^{2}\right) F(r)$ and $\left(1-r^{2}\right) \hat{F}(r)$ when $F(r)=(1-A r) /(1-B r)=\varphi_{A, B}(r)$ for $A=0.7$ and $B=-0.3$ (cf. Lemma 4.2). We now prove the above fact in the general case.

Proposition 2.4. For a continuous function $F$ on the interval $[0,1)$, the maximal function $\hat{F}$ satisfies

$$
\lim _{r \rightarrow 1-}\left(1-r^{2}\right) \hat{F}(r)=\sup _{0 \leqslant r<1}\left(1-r^{2}\right) \hat{F}(r)=\sup _{0 \leqslant r<1}\left(1-r^{2}\right)|F(r)| .
$$

Proof. By (2.8), we obtain

$$
\limsup _{r \rightarrow 1-}\left(1-r^{2}\right) \hat{F}(r) \leqslant \sup _{0 \leqslant r<1}\left(1-r^{2}\right) \hat{F}(r)=\sup _{0 \leqslant r<1}\left(1-r^{2}\right)|F(r)| .
$$

It remains to prove the opposite direction. Let $M$ be an arbitrary number with $M<$ $\sup _{0 \leqslant r<1}\left(1-r^{2}\right)|F(r)|$. It is enough to prove the inequality $M<\lim _{r \rightarrow 1-}\left(1-r^{2}\right) \hat{F}(r)$. 
By the choice of $M$, we can find a number $r_{1}$ in $[0,1)$ so that $M<\left(1-r_{1}^{2}\right)\left|F\left(r_{1}\right)\right|$ holds. Then, for any $r \in\left(r_{1}, 1\right)$, we have

$$
\begin{aligned}
\left(1-r^{2}\right) \hat{F}(r) & =\left(1-r^{2}\right) \max _{0 \leqslant s \leqslant r} K(r, s)|F(s)| \\
& \geqslant\left(1-r^{2}\right) K\left(r, r_{1}\right)\left|F\left(r_{1}\right)\right| \\
& >M \frac{1-r^{2}}{1-r_{1}^{2}} K\left(r, r_{1}\right) \\
& =M \frac{r_{1}\left(1-r^{2}\right)+r^{2}-r_{1}^{2}}{r\left(1-r_{1}^{2}\right)} .
\end{aligned}
$$

We take the lower limit as $r \rightarrow 1-$ to obtain the inequality $\liminf _{r \rightarrow 1-}\left(1-r^{2}\right) \hat{F}(r) \geqslant M$. Letting $M$ tend to $\sup _{0 \leqslant r<1}\left(1-r^{2}\right)|F(r)|$, we have

$$
\liminf _{r \rightarrow 1-}\left(1-r^{2}\right) \hat{F}(r) \geqslant \sup _{0 \leqslant r<1}\left(1-r^{2}\right)|F(r)| .
$$

Hence, the limit of $\left(1-r^{2}\right) \hat{F}(r)$ exists when $r$ tends to 1 from the left and it equals the supremum of $\left(1-r^{2}\right)|F(r)|$ over $0 \leqslant r<1$.

As a corollary, we note the following simple fact.

Corollary 2.5. For $F, G \in C([0,1))$,

$$
\sup _{0 \leqslant r<1}\left(1-r^{2}\right)(\hat{F}(r)+\hat{G}(r))=\sup _{0 \leqslant r<1}\left(1-r^{2}\right) \hat{F}(r)+\sup _{0 \leqslant r<1}\left(1-r^{2}\right) \hat{G}(r) .
$$

\section{Proof of the main theorem}

For $\varphi \in \mathcal{M}$, we define the functions $h_{\varphi}$ and $k_{\varphi}$ in $\mathcal{A}$ by the relations

$$
\frac{z h_{\varphi}^{\prime}(z)}{h_{\varphi}(z)}=\varphi(z) \quad \text { and } \quad 1+\frac{z k_{\varphi}^{\prime \prime}(z)}{k_{\varphi}^{\prime}(z)}=\varphi(z)
$$

i.e.

$$
h_{\varphi}(z)=z \exp \int_{0}^{z} \frac{\varphi(t)-1}{t} \mathrm{~d} t \quad \text { and } \quad k_{\varphi}(z)=\int_{0}^{z}\left(\exp \int_{0}^{\zeta} \frac{\varphi(t)-1}{t} \mathrm{~d} t\right) \mathrm{d} \zeta .
$$

For instance, we can compute $h_{\varphi_{A, B}}$ and $k_{\varphi_{A, B}}$ for $-1 \leqslant B<A \leqslant 1$ as follows:

$$
h_{\varphi_{A, B}}(z)=z k_{\varphi_{A, B}}^{\prime}(z)= \begin{cases}z(1+B z)^{(A-B) / B}, & B \neq 0, \\ z \mathrm{e}^{A z}, & B=0,\end{cases}
$$

and

$$
k_{\varphi_{A, B}}(z)= \begin{cases}(1 / A)\left((1+B z)^{A / B}-1\right), & A \neq 0, B \neq 0 \\ (1 / B) \log (1+B z), & A=0, \\ (1 / A)\left(\mathrm{e}^{A z}-1\right), & B=0 .\end{cases}
$$


Under some additional assumptions on $\varphi$, Ma and Minda showed $[\mathbf{9}]$ that these functions are extremal in $\mathcal{S}^{*}(\varphi)$ and $\mathcal{K}(\varphi)$, respectively, in many respects. In particular, they obtain the following lemma. In order to clarify what assumptions are necessary for $\varphi$, we will also reproduce the proof of the lemma.

Lemma 3.1 (see Theorem 1 in [9]). Suppose that a function $\varphi \in \mathcal{M}$ is univalent and $\varphi(\mathbb{D})$ is starlike with respect to 1 . Then $f^{\prime} \prec k_{\varphi}^{\prime}$ holds for every $f \in \mathcal{K}(\varphi)$.

Proof. Let $g=c \log k_{\varphi}^{\prime}$, where $c=1 / \varphi^{\prime}(0)$. Since $c(\varphi-1) \in \mathcal{A}$ is starlike, we can see that

$$
1+\frac{z g^{\prime}(z)}{g^{\prime \prime}(z)}=\frac{z \varphi^{\prime}(z)}{\varphi(z)-1}
$$

has positive real part; in other words, $g$ is convex. By assumption, the relation $c z f^{\prime \prime} / f^{\prime} \prec$ $c(\varphi-1)=c z k_{\varphi}^{\prime \prime} / k_{\varphi}^{\prime}=z g^{\prime}$ holds. By Suffridge's theorem [12, Theorem 3], one obtains $c \log f^{\prime} \prec g=c \log k_{\varphi}^{\prime}$, and, hence, $f^{\prime} \prec k_{\varphi}^{\prime}$. (Recall that convexity of $g$ was essential in this theorem.)

In general, for $f, g \in \mathcal{A}$, the condition $f^{\prime} \prec g^{\prime}$ implies the inequality $\left\|T_{f}\right\| \leqslant\left\|T_{g}\right\|$ (see $[\mathbf{6}]$ ). Hence, we obtain the following as a corollary.

Theorem 3.2. Let $\varphi$ be as in Lemma 3.1. If $f \in \mathcal{K}(\varphi)$, then $\left\|T_{f}\right\| \leqslant\left\|T_{k_{\varphi}}\right\|$ holds, where $k_{\varphi}$ is the function given in (3.2).

We now prove Theorem 1.1. It is convenient below to introduce the class $\mathcal{B}$ of analytic functions $\omega$ on the unit disc with $|\omega(z)| \leqslant|z|$. Let $f \in \mathcal{C}(\varphi, \psi)$. Then, by definition, there is a function $h \in \mathcal{K}(\varphi)$ such that $f^{\prime} / h^{\prime} \prec \psi$. By Lemma 3.1, we see that $h^{\prime} \prec k_{\varphi}^{\prime}$. Let $\omega_{1}$ and $\omega_{2}$ be analytic functions in $\mathcal{B}$ satisfying $h^{\prime}=k_{\varphi}^{\prime} \circ \omega_{1}$ and $f^{\prime} / h^{\prime}=\psi \circ \omega_{2}$. Conversely, for any pair of functions $\omega_{1}, \omega_{2} \in \mathcal{B}$, the function $f$ is uniquely determined so that the above relations hold. We occasionally write $f=f\left[\omega_{1}, \omega_{2}\right]$. By taking the logarithmic derivative, these relations yield

$$
\begin{aligned}
T_{f} & =T_{h}+\frac{\left(\psi^{\prime} \circ \omega_{2}\right) \omega_{2}^{\prime}}{\psi \circ \omega_{2}} \\
& =\frac{\left(\varphi \circ \omega_{1}-1\right) \omega_{1}^{\prime}}{\omega_{1}}+\frac{\left(\psi^{\prime} \circ \omega_{2}\right) \omega_{2}^{\prime}}{\psi \circ \omega_{2}} \\
& =\omega_{1}^{\prime}\left(\Phi \circ \omega_{1}\right)+\omega_{2}^{\prime}\left(\Psi \circ \omega_{2}\right),
\end{aligned}
$$

where we have set $\Phi(z)=(\varphi(z)-1) / z$ and $\Psi(z)=\psi^{\prime}(z) / \psi(z)$.

For an analytic function $g$ on $\mathbb{D}$, we will denote by $\hat{M}(r, g)$ the maximal function of $M(r, g)=\max \{|g(z)|:|z|=r\}$.

Fix a point $z_{0} \in \mathbb{D}$ with $r=\left|z_{0}\right|>0$. For any pair of points $w_{1}, w_{2}$ with $r_{j}=\left|w_{j}\right| \leqslant r$, consider functions $\omega_{1}, \omega_{2} \in \mathcal{B}$ with $\omega_{j}\left(z_{0}\right)=w_{j}$ for $j=1,2$. By $(2.2)$, we observe that

$$
\begin{aligned}
\left|T_{f\left[\omega_{1}, \omega_{2}\right]}\left(z_{0}\right)\right| & \leqslant K\left(r, r_{1}\right)\left|\Phi\left(w_{1}\right)\right|+K\left(r, r_{2}\right)\left|\Psi\left(w_{2}\right)\right| \\
& \leqslant K\left(r, r_{1}\right) M\left(r_{1}, \Phi\right)+K\left(r, r_{2}\right) M\left(r_{2}, \Psi\right) \\
& \leqslant \hat{M}(r, \Phi)+\hat{M}(r, \Psi) .
\end{aligned}
$$


Hence, by Proposition 2.4 and its corollary,

$$
\begin{aligned}
\left\|T_{f}\right\| & \leqslant \sup _{0 \leqslant r<1}\left(1-r^{2}\right)(\hat{M}(r, \Phi)+\hat{M}(r, \Psi)) \\
& =\sup _{0 \leqslant r<1}\left(1-r^{2}\right) M(r, \Phi)+\sup _{0 \leqslant r<1}\left(1-r^{2}\right) M(r, \Psi) .
\end{aligned}
$$

Thus (1.3) has been proved.

Next we demonstrate the sharpness under the additional assumption (1.4). For a given $0 \leqslant r<1$, we choose $r_{1}, r_{2} \in[0, r]$ so that $\hat{M}(r, \Phi)=K\left(r, r_{1}\right) M(r, \Phi)$ and $\hat{M}(r, \Psi)=$ $K\left(r, r_{2}\right) M(r, \Psi)$. For each $j=1,2$, let $\omega_{j}$ be the function of the form (2.1) with $w_{0}=\varepsilon r_{j}$ and $\lambda=\varepsilon\left|z_{0}\right|^{2} / z_{0}^{2}$. Then equality holds at each step of the estimations in (3.5). Hence,

$$
\max _{f \in \mathcal{C}(\varphi, \psi)} M\left(T_{f}, r\right)=\hat{M}(r, \Phi)+\hat{M}(r, \Psi)
$$

holds for each $r<1$. We remark that the extremal function attaining the above maximum is uniquely determined for each $r<1$. Now it is evident that the estimate (1.3) is best possible if (1.4) is satisfied.

\section{Applications to the class $\mathcal{C}\left(\varphi_{A_{1}, B_{1}}, \varphi_{A_{2}, B_{2}}\right)$}

As an application of Theorem 1.1, we consider the case when $\varphi=\varphi_{A_{1}, B_{1}}$ and $\psi=\varphi_{A_{2}, B_{2}}$ for some real numbers $A_{1}, B_{1}, A_{2}, B_{2}$ with $-1 \leqslant A_{j}<B_{j} \leqslant 1$ for $j=1,2$, where $\varphi_{A, B}$ is the function given in (1.1).

It is convenient to have the exact value of

$$
E(A, B)=\sup _{|z|<1} \frac{1-|z|^{2}}{|1+A z||1+B z|}
$$

for $-1 \leqslant B<A \leqslant 1$. To this end, we prepare the next elementary lemma.

Lemma 4.1. For real numbers $A, B$ with $-1 \leqslant B<A \leqslant 1$, the inequality

$$
|1+A z||1+B z| \geqslant(1+\varepsilon A|z|)(1+\varepsilon B|z|)
$$

holds for every $z \in \mathbb{D}$. Here, $\varepsilon=1$ when $A+B \leqslant 0$ and $\varepsilon=-1$ when $A+B \geqslant 0$.

Proof. First assume that $A+B \leqslant 0$. If $A B \geqslant 0$, then $A \leqslant 0$ and $B \leqslant 0$, and, thus, the claim is obvious. If $A B<0$, the assumptions imply $B<0<A$ and

$$
\begin{aligned}
\min _{|z|=r}|1+A z|^{2}|1+B z|^{2} & =\min _{-r \leqslant x \leqslant r}\left(1+A^{2} r^{2}+2 A x\right)\left(1+B^{2} r^{2}+2 B x\right) \\
& =(1-A r)^{2}(1-B r)^{2} .
\end{aligned}
$$

Hence, the required inequality follows. The other case when $A+B \geqslant 0$ can be treated similarly.

We are now ready to compute the value of $E(A, B)$. 
Lemma 4.2. If $-1 \leqslant B<A \leqslant 1$, then

$$
E(A, B)=\frac{2}{1-A B+\sqrt{\left(1-A^{2}\right)\left(1-B^{2}\right)}} .
$$

Proof. First we assume that $A+B \geqslant 0$. Then, by Lemma 4.1, we obtain the expression

$$
E(A, B)=\sup _{0 \leqslant r<1} g(r)
$$

where we set

$$
g(x)=\frac{1-x^{2}}{(1-A x)(1-B x)} .
$$

A simple calculation gives $E(A, B)=g\left(x_{0}\right)$, where $x_{0}$ is the unique zero of $g^{\prime}(x)$ in $0 \leqslant x<1$, that is,

$$
x_{0}=\frac{A+B}{1+A B+\sqrt{\left(1-A^{2}\right)\left(1-B^{2}\right)}} .
$$

Noting the relation

$$
(A+B) x_{0}^{2}-2(1+A B) x_{0}-(A+B)=0,
$$

we get (4.2). The case when $A+B<0$ can be reduced to the previous one by using the obvious relation $E(A, B)=E(-B,-A)$. The proof is now complete.

As an immediate consequence of this together with Theorem 3.2, we obtain the following theorem.

Theorem 4.3. Let $-1 \leqslant B<A \leqslant 1$. If $f \in \mathcal{K}\left(\varphi_{A, B}\right)$, then

$$
\left\|T_{f}\right\| \leqslant \frac{2(A-B)}{1+\sqrt{1-B^{2}}},
$$

and equality holds when $f=k_{\varphi_{A, B}}$.

Proof. If $f \in \mathcal{K}\left(\varphi_{A, B}\right)$, by Theorem 3.2, we have

$$
\left\|T_{f}\right\| \leqslant\left\|T_{k}\right\|
$$

where $k$ denotes the function $k_{\varphi_{A, B}}$ given in (3.4). Since

$$
\frac{k^{\prime \prime}(z)}{k^{\prime}(z)}=\frac{\varphi_{A, B}(z)-1}{z}=\frac{A-B}{1+B z}
$$

we obtain

$$
\left\|T_{k}\right\|=(A-B) E(0, B)=\frac{2(A-B)}{1+\sqrt{1-B^{2}}}
$$

by Lemma 4.2 . 
Noting the expressions

$$
\frac{\varphi_{A, B}(z)-1}{z}=\frac{A-B}{1+B z} \quad \text { and } \quad \frac{\varphi_{A, B}^{\prime}(z)}{\varphi_{A, B}(z)}=\frac{A-B}{(1+A z)(1+B z)}
$$

and using Lemma 4.1, we see that the condition (1.4) is fulfilled for $\varphi=\varphi_{A_{1}, B_{1}}$ and $\psi=\varphi_{A_{2}, B_{2}}$ if either

$$
B_{1} \leqslant 0 \quad \text { and } \quad A_{2}+B_{2} \leqslant 0 \quad(\text { with } \varepsilon=1)
$$

or

$$
\left.B_{1} \geqslant 0 \text { and } A_{2}+B_{2} \geqslant 0 \quad \text { (with } \varepsilon=-1\right) .
$$

Theorem 1.1 together with Lemma 4.2 now yields the following result.

Theorem 4.4. Let $-1 \leqslant B_{j}<A_{j} \leqslant 1$ for $j=1$, 2. If $f \in \mathcal{C}\left(\varphi_{A_{1}, B_{1}}, \varphi_{A_{2}, B_{2}}\right)$, then

$$
\left\|T_{f}\right\| \leqslant \frac{2\left(A_{1}-B_{1}\right)}{1+\sqrt{1-B_{1}^{2}}}+\frac{2\left(A_{2}-B_{2}\right)}{1-A_{2} B_{2}+\sqrt{\left(1-A_{2}^{2}\right)\left(1-B_{2}^{2}\right)}} .
$$

The inequality is sharp when $B_{1}\left(A_{2}+B_{2}\right) \geqslant 0$.

The second author gave the inequality

$$
\left\|T_{f}\right\| \leqslant 6 k
$$

for functions $f \in \mathcal{S}^{*}\left(\varphi_{-k, k}\right)$ for $0 \leqslant k \leqslant 1$ in [13, Theorem 4.3]. The following corollary improves the above estimate.

Corollary 4.5. For $0 \leqslant k \leqslant 1$, functions $f \in \mathcal{S}^{*}\left(\varphi_{-k, k}\right)$ satisfy the inequality

$$
\left\|T_{f}\right\| \leqslant \frac{4 k}{1+\sqrt{1-k^{2}}}+2 k .
$$

Proof. Since $\mathcal{S}^{*}\left(\varphi_{-k, k}\right) \subset \mathcal{C}\left(\varphi_{-k, k}, \varphi_{-k, k}\right)$, the above inequality follows from Theorem 4.4 with $A_{j}=k, B_{j}=-k$.

Note that the estimate in the corollary may not be sharp, though it is sharp for the $\operatorname{class} \mathcal{C}\left(\varphi_{-k, k}, \varphi_{-k, k}\right)$.

\section{Relationship with the Hardy space}

The Hardy space $\mathcal{H}^{p}(0<p \leqslant \infty)$ is the class of all functions $f$ analytic in $\mathbb{D}$ such that

$$
\|f\|_{p}:=\lim _{r \rightarrow 1^{-}} M_{p}(r, f)<\infty
$$

where

$$
M_{p}(r, f)= \begin{cases}\left(\frac{1}{2 \pi} \int_{0}^{2 \pi}\left|f\left(r \mathrm{e}^{\mathrm{i} \theta}\right)\right|^{p} \mathrm{~d} \theta\right)^{1 / p}, & 0<p<\infty \\ M(r, f)=\max _{|z| \leqslant r}|f(z)|, & p=\infty\end{cases}
$$


Let BMOA be the family of functions $f$ analytic in $\mathbb{D}$ with finite BMOA norm:

$$
\|f\|_{*}:=\sup _{\alpha \in \mathbb{D}}\left\|f_{\alpha}\right\|_{2}+|f(0)|<\infty
$$

where $f_{\alpha}(z)=f((z+\alpha) /(1+\bar{\alpha} z))-f(\alpha)$. Note that $\mathcal{H}^{\infty} \subset$ BMOA $\subset \bigcap_{0<p<\infty} \mathcal{H}^{p}$. See [1] and [2] for further information.

A simple relationship between the class $\mathcal{C}(\varphi, \psi)$ and the Hardy space $\mathcal{H}^{p}$ is given by the following theorem.

Theorem 5.1. Let $1 \leqslant p<\infty$. Suppose that $\varphi \in \mathcal{M}$ is univalent, $\varphi(\mathbb{D})$ is starlike with respect to 1 and $k_{\varphi}^{\prime} \in \mathcal{H}^{1}$, where $k_{\varphi}$ is given by (3.2). Then $\mathcal{C}(\varphi, \psi) \subset \mathcal{H}^{p}$ for every $\psi \in \mathcal{M} \cap \mathcal{H}^{p}$.

Proof. If $f \in \mathcal{C}(\varphi, \psi)$, from (1.2) we have

$$
f(z)=\int_{0}^{z} h^{\prime}(t) \psi(\omega(t)) \mathrm{d} t
$$

where $h \in \mathcal{K}(\varphi)$ and $|\omega(z)| \leqslant|z|$. By Littlewood's subordination theorem [2, Theorem 1.7], it follows that $\psi \circ \omega \in \mathcal{H}^{p}$ for $\psi \in \mathcal{M} \cap \mathcal{H}^{p}$. By assumption, $h^{\prime} \prec k_{\varphi}^{\prime} \in \mathcal{H}^{1}$, and hence $h^{\prime} \in \mathcal{H}^{1}$. This implies that $h \in \mathcal{H}^{\infty} \subset$ BMOA. Now the following theorem yields the desired result.

Theorem 5.2 (Aleman and Siskakis [1]). Let $h$ be an analytic function in the unit disc and let $1 \leqslant p<\infty$. The operator

$$
f \mapsto \frac{1}{z} \int_{0}^{z} f(t) h^{\prime}(t) \mathrm{d} t
$$

maps $\mathcal{H}^{p}$ continuously into itself if and only if $h \in \mathrm{BMOA}$.

Corollary 5.3. Let $-1 \leqslant B<A \leqslant 1$. If $-1<B$ or $A \leqslant 0$, then, for any number $1 \leqslant p<\infty$, the relation $\mathcal{C}\left(\varphi_{A, B}, \psi\right) \subset \mathcal{H}^{p}$ holds for all $\psi \in \mathcal{M} \cap \mathcal{H}^{p}$. If $B=-1$ and $A>0$, then, for each $1 \leqslant p<\infty$, there exists a function $\psi \in \mathcal{M} \cap \mathcal{H}^{p}$ such that the relation $\mathcal{C}\left(\varphi_{A, B}, \psi\right) \subset \mathcal{H}^{p}$ does not hold.

Proof. In view of (3.3), we can see that $k_{\varphi_{A, B}}^{\prime} \in \mathcal{H}^{1}$ if and only if $-1<B$ or $A<0$. Thus, by Theorem 5.1, the statement holds in this case. When $B=-1$ and $A=0$, $\varphi(z)=\varphi_{0,-1}(z)=1 /(1-z)$, therefore $k_{\varphi}^{\prime}(z)=1 /(1-z)$. If $h^{\prime} \prec k_{\varphi}^{\prime}$, then $h^{\prime}=1 /(1-\omega)$, where $\omega: \mathbb{D} \rightarrow \mathbb{D}$ is analytic with $\omega(0)=0$. Hence,

$$
\left(1-|z|^{2}\right)\left|h^{\prime}(z)\right| \leqslant \frac{1-|z|^{2}}{1-|\omega(z)|} \leqslant 1+|z|<2,
$$

which implies $h \in$ BMOA because a univalent Bloch function is known to belong to BMOA. Now the theorem of Aleman and Siskakis implies the desired claim even in this case. 
Now suppose $B=-1$ and $A>0$. Let $p_{0} \in[1, \infty)$ be given. Choose a number $C$ so that

$$
\max \left\{\frac{1}{p_{0}}-A, 0\right\} \leqslant C<\frac{1}{p_{0}}
$$

and set $\psi(z)=(1-z)^{-C}$. Note first that

$$
\psi \in \bigcap_{0<p<1 / C} \mathcal{H}^{p} \subset \mathcal{H}^{p_{0}} .
$$

Then the function $f \in \mathcal{A}$ determined by

$$
f^{\prime}(z)=k_{\varphi_{A,-1}}^{\prime}(z) \psi(z)=(1-z)^{-A-C-1}
$$

belongs to the class $\mathcal{C}\left(\varphi_{A,-1}, \psi\right)$. In view of the form

$$
f(z)=\frac{(1-z)^{-A-C}-1}{A+C}
$$

of $f$, we see that $f$ does not belong to $\mathcal{H}^{p}$ for $p \geqslant 1 /(A+C)$. Since $p_{0} \geqslant 1 /(A+C)$ by the choice of $C$, we conclude that $f \in \mathcal{C}\left(\varphi_{A,-1}, \psi\right) \backslash \mathcal{H}^{p_{0}}$.

Remark 5.4. In general, if $\psi \in \mathcal{M}$ has positive real part, by [2, Theorem 3.2], we have

$$
\psi \in \bigcap_{0<p<1} \mathcal{H}^{p}
$$

We also note that

$$
\mathcal{C}(\varphi, \psi) \subset \mathcal{C} \subset \mathcal{S} \subset \bigcap_{0<p<1 / 2} \mathcal{H}^{p}
$$

for $\varphi \in \mathcal{M}$ with $\operatorname{Re} \varphi>0$ and $\psi \in \mathcal{M}$ with $\operatorname{Re} \mathrm{e}^{\mathrm{i} \gamma} \psi>0$ for some $\gamma \in \mathbb{R}$ (see $[\mathbf{2}$, Theorem 3.16]). The above ranges for $p$ are sharp.

Acknowledgements. Y.C.K. was supported by the Korea Basic Science Research Foundation, under grant no. DP0022. T.S. was partly supported by the Ministry of Education, Grant-in-Aid for Encouragement of Young Scientists, 9740056 and 14740100.

\section{References}

1. A. Aleman and A. G. Siskakis, An integral operator on $H^{p}$, Complex Variables Theory Applic. 28 (1995), 149-158.

2. P. L. Duren, Theory of $H^{p}$ spaces (Academic, 1970).

3. P. L. Duren, Univalent functions (Springer, 1983).

4. W. JANowsKI, Some extremal problems for certain families of analytic functions, I, Ann. Polon. Math. 23 (1973), 159-177.

5. W. Janowski, Some extremal problems for certain families of analytic functions, II, Bull. Acad. Polon. Sci. Ser. Sci. Math. Astronom. Phys. 21 (1973), 17-25.

6. Y. C. Kim And T. Sugawa, Growth and coefficient estimates for uniformly locally univalent functions on the unit disk, Rocky Mt. J. Math. 32 (2002), 179-200. 
7. Y. C. KIM AND T. SugAWA, A conformal invariant for non-vanishing analytic functions and its applications, Michigan Math. J., in press.

8. Y. C. Kim, J. H. Choi And T. Sugawa, Coefficient bounds and convolution properties for certain classes of close-to-convex functions, Proc. Jpn Acad. A 76 (2000), 95-98.

9. W. MA AND D. MindA, A unified treatment of some special classes of univalent functions, in Proc. Conf. on Complex Analysis, Tianjin, 1992 (ed. Z. Li, F. Ren, L. Yang and S. Zhang), pp. 157-169 (International Press, Cambridge, MA, 1992).

10. Y. OKUYAMA, The norm estimates of pre-Schwarzian derivatives of spiral-like functions, Complex Variables Theory Applic. 42 (2000), 225-239.

11. H. Silverman And E. M. Silvia, Subclasses of starlike functions subordinate to convex functions, Can. J. Math. 37 (1985), 48-61.

12. T. J. Suffridge, Some remarks on convex maps of the unit disk, Duke Math. J. 37 (1970), 775-777.

13. T. Sugawa, Holomorphic motions and quasiconformal extensions, Ann. Univ. Mariae Curie-Sktodowska A 53 (1999), 239-252.

14. S. YAmAshitA, Gelfer functions, integral means, bounded mean oscillation, and univalency, Trans. Am. Math. Soc. 321 (1990), 245-259.

15. S. YAmashita, Norm estimates for function starlike or convex of order alpha, Hokkaido Math. J. 28 (1999), 217-230.

16. I. V. Zhuravlev, Model of the universal Teichmüller space, Sb. Math. J. 27 (1986), 691-697. 